\title{
CONSTRUCTION OF POLYNOMIAL POLYTOPES BY USING SEGMENT LEMMA
}

\author{
Sayed Abdul Hannan Sadat (D), Vakıf Cafer (Dzhafarov) (D), Taner Büyükköroğlu* \\ Department of Mathematics, Faculty of Science, Eskişehir Technical University, Turkey \\ tbuyukkoroglu@eskisehir.edu.tr (*corresponding author)
}

\begin{abstract}
A polynomial with all roots lying in the open left half plane of the complex plane is called Hurwitz stable. The convex hull of a finite number of polynomials of the same order is called a polynomial polytope. By the Edge theorem, a polynomial polytope with the invariant degree is Hurwitz stable if and only if all edges are Hurwitz stable. Due to the Edge Theorem, the segment stability criterions are of great importance. In this paper, we consider a construction of stable polytopes by using the Segment Lemma. It is constructed stable polytopes with nonzero volumes. The obtained results can be used, for example, in the stabilization problems of unstable transfer functions by lower-order controllers.
\end{abstract}

Keywords: Polynomial, Hurwitz stability, Segment lemma, polynomial polytope.

\section{Introduction}

Let the following $n$-order polynomial

$p(s)=a_{n} s^{n}+a_{n-1} s^{n-1}+\cdots+a_{1} s+a_{0} \quad\left(a_{n}>0\right)$

be given. The polynomial (1) has $n$ roots $s_{1}, s_{2}, \ldots, s_{n}$ and if $\operatorname{Re}\left(s_{i}\right)<0$ for all $i=1,2, \ldots, n$ then it is called Hurwitz stable. From now we will use the term "stable" instead of "Hurwitz stable".

A necessary condition for stability of (1) is the positivity of all coefficients $a_{i}(i=0,1, \ldots, n)$. This necessary condition is not sufficient, unless $n \leq 2$.

It is well-known that the set of all $n$-order stable polynomials is open and nonconvex. Nonconvexity means that given two stable $n$-order polynomials $a_{1}(s)$ and $a_{2}(s)$ their convex combination set $\left\{(1-\lambda) a_{1}(s)+\lambda a_{2}(s): \lambda \in[0,1]\right\}$ may contain an unstable polynomial. 
The above convex combination set is called the polynomial segment connecting $a_{1}(s)$ and $a_{2}(s)$ and is denoted by $\left[a_{1}(s), a_{2}(s)\right]$.

Let $n$-order polynomials $a_{1}(s), \ldots, a_{k}(s)$ be given. Then the set

$\operatorname{conv}\left\{a_{1}(s), \ldots, a_{k}(s)\right\}$

is called a polynomial polytope, where "conv" stands for the convex hull. Given the polytope (2) the polynomials $a_{i}(s)$ are called the generators, and the segments $\left[a_{i}(s), a_{j}(s)\right]$ are called the edges of the polytope (2) $(i<j, i, j=1,2, \ldots, k)$. The polytope (2) is called stable if all polynomials from (2) are stable.

By the Edge Theorem [1] given $n$-order polynomial polytope (2) with invariant degree and at least one stable member is stable if and only if all its edges are stable.

Due to the Edge Theorem the segment stability criterions are of great importance. This problem has been considered in the works [2-8].

The following Segment Lemma is one of well-known criterion for segment stability.

For the polynomial (1) write $a(s)=a^{e}(s)+s a^{o}(s)$, where $a^{e}(s)$ and $a^{o}(s)$ contain only even degrees and set $\delta^{e}(\omega)=a^{e}(j \omega), \delta^{o}(\omega)=a^{o}(j \omega)\left(j^{2}=-1, \omega>0\right)$.

Lemma 1.1 (Segment Lemma, [2, page 80]) Let $a_{1}(s)$ and $a_{2}(s)$ be $n$-order stable polynomials with positive coefficients. Then the segment $\left[a_{1}(s), a_{2}(s)\right]$ is stable $\Leftrightarrow$ There is no $\omega>0$ satisfying the relations

$\delta_{1}^{e}(\omega) \delta_{2}^{o}(\omega)-\delta_{2}^{e}(\omega) \delta_{1}^{o}(\omega)=0$,

$\delta_{1}^{e}(\omega) \delta_{2}^{e}(\omega) \leq 0$

$\delta_{1}^{o}(\omega) \delta_{2}^{o}(\omega) \leq 0$.

The following lemma is a consequence of Lemma 1.1 .

Lemma 1.2 Let $a_{1}(s)$ and $a_{2}(s)$ be $n$-order stable polynomials with positive coefficients and the open segment $\left(a_{1}(s), a_{2}(s)\right)$ contains at least one stable member. Then the open segment $\left(a_{1}(s), a_{2}(s)\right)$ is stable $\Leftrightarrow$ There is no $\omega>0$ satisfying (3), (4) and (5).

The geometrical and topological properties of stable polynomials have been studied in many works (see [9-13]). This paper continues these studies. Here we construct stable polynomial polytopes by using the Edge Theorem and Lemmas 1.1 and 1.2. As a starting point we use a factorized stable polynomial and specially defined $n$ boundary polynomials. The convex hull of these $n+1$ points becomes stable polytope.

The following criterions are well-known [3]:

A fourth order polynomial $a_{4} s^{4}+a_{3} s^{3}+a_{2} s^{2}+a_{1} s+a_{0}$ with positive coefficients is stable if and only if

$$
a_{1} a_{2} a_{3}-a_{1}^{2} a_{4}-a_{0} a_{3}^{2}>0
$$


A third order polynomial $a_{3} s^{3}+a_{2} s^{2}+a_{1} s+a_{0}$ with positive coefficints is stable if and only if $a_{1} a_{2}-a_{0} a_{3}>0$.

\section{Construction of stable polytopes}

In this section we construct stable polytopes with nonzero volumes. To do this we choose any factorized stable polynomial and consider polynomial segments starting from this polynomial and ending on the stability boundary.

Consider stable polynomials

$$
p(s)=\left(s^{2}+b_{m} s+c_{m}\right) \cdot \cdots \cdot\left(s^{2}+b_{1} s+c_{1}\right)
$$

if $n=2 m$ and

$$
p(s)=\left(s^{2}+b_{m-1} s+c_{m-1}\right) \cdot \cdots \cdot\left(s^{2}+b_{1} s+c_{1}\right) \cdot\left(s+b_{m}\right)
$$

if $n=2 m-1$. Here $b_{i}>0, c_{i}>0(i=1,2, \ldots, m)$. Consider $n$ polynomials

$$
\begin{gathered}
p_{1}(s)=\left(s^{2}+c_{m}\right) \cdot\left(s^{2}+b_{m-1} s+c_{m-1}\right) \cdot \cdots \cdot\left(s^{2}+b_{1} s+c_{1}\right) \\
p_{2}(s)=\left(s^{2}+b_{m} s\right) \cdot\left(s^{2}+b_{m-1} s+c_{m-1}\right) \cdots \cdot\left(s^{2}+b_{1} s+c_{1}\right) \\
\vdots \\
p_{n}(s)=\left(s^{2}+b_{m-1} s+c_{m-1}\right) \cdot\left(s^{2}+b_{m-1} s+c_{m-1}\right) \cdots \cdot\left(s^{2}+b_{1} s\right)
\end{gathered}
$$

if $n=2 m$ and

$$
\begin{gathered}
p_{1}(s)=\left(s^{2}+c_{m-1}\right) \cdot \cdots \cdot\left(s^{2}+b_{1} s+c_{1}\right) \cdot\left(s+b_{m}\right) \\
p_{2}(s)=\left(s^{2}+b_{m-1} s\right) \cdot \cdots \cdot\left(s^{2}+b_{1} s+c_{1}\right) \cdot\left(s+b_{m}\right) \\
\vdots \\
p_{n}(s)=\left(s^{2}+b_{m} s+c_{m}\right) \cdots \cdot\left(s^{2}+b_{1} s+c_{1}\right) \cdot s
\end{gathered}
$$

if $n=2 m-1$. The polynomials $p_{1}(s), \ldots, p_{n}(s)$ belong to the stability boundary. Consider the polytope

$\mathcal{P}=\operatorname{conv}\left\{p(s), p_{1}(s), \ldots, p_{n}(s)\right\}$

The polytope (6) has totally $1+2+3+\cdots n=\frac{n(n+1)}{2}$ edges and if all edges are stable then by the Edge Theorem the polytope (6) is stable. Some edges such as $\left[p(s), p_{i}(s)\right)(i=$ $1,2, \ldots, n)$ are stable by the construction.

Due to the factorization, the following lemmas can be used for proving the stability of the polytope (6).

Lemma 2.1 Consider the following polynomials 


$$
\begin{aligned}
& q(s)=\left(s^{2}+\alpha_{1} s+\alpha_{2}\right) \cdot\left(s^{2}+\alpha_{3} s+\alpha_{4}\right), \\
& q_{1}(s)=\left(s^{2}+\alpha_{2}\right) \cdot\left(s^{2}+\alpha_{3} s+\alpha_{4}\right), \\
& q_{2}(s)=\left(s^{2}+\alpha_{1} s\right) \cdot\left(s^{2}+\alpha_{3} s+\alpha_{4}\right), \\
& q_{3}(s)=\left(s^{2}+\alpha_{1} s+\alpha_{2}\right) \cdot\left(s^{2}+\alpha_{4}\right), \\
& q_{4}(s)=\left(s^{2}+\alpha_{1} s+\alpha_{2}\right) \cdot\left(s^{2}+\alpha_{3} s\right)
\end{aligned}
$$

with $\alpha_{i}>0(i=1,2,3,4)$. Then

1) The segments $\left[q(s), q_{i}(s)\right),\left(q_{1}(s), q_{2}(s)\right),\left(q_{3}(s), q_{4}(s)\right)$ are stable,

2) The segment $\left(q_{1}(s), q_{3}(s)\right)$ is stable in the case $\alpha_{2} \neq \alpha_{4}$ and belongs to the stability boundary in the case $\alpha_{2}=\alpha_{4}$,

3) The segment $\left[q_{2}(s), q_{4}(s)\right]$ belongs to the stability boundary.

\section{Proof.}

1) Stability of the segments $\left[q(s), q_{i}(s)\right),\left(q_{1}(s), q_{2}(s)\right),\left(q_{3}(s), q_{4}(s)\right)$ are obvious $(i=1,2,3,4)$.

2) Consider the segment $\left(q_{1}(s), q_{3}(s)\right)$. For any $\lambda \in(0,1)$ we have

$$
\begin{array}{r}
(1-\lambda) q_{1}(s)+\lambda q_{3}(s)=s^{4}+\left(\lambda \alpha_{1}-\lambda \alpha_{3}+\alpha_{3}\right) s^{3}+\left(\alpha_{2}+\alpha_{4}\right) s^{2}+ \\
\left(\lambda \alpha_{1} \alpha_{4}-\lambda \alpha_{2} \alpha_{3}+\alpha_{2} \alpha_{3}\right) s+\alpha_{2} \alpha_{4}
\end{array}
$$

and (see Introduction)

$$
a_{1} a_{2} a_{3}-a_{1}^{2} a_{4}-a_{0} a_{3}^{2}=\lambda \alpha_{1} \alpha_{3}\left(\alpha_{2}-\alpha_{4}\right)(1-\lambda) \geq 0 .
$$

Therefore the segment $\left(q_{2}(s), q_{3}(s)\right)$ either is stable or belongs to the stability boundary.

3) Consider the segment $\left(q_{2}(s), q_{4}(s)\right)$. For any $\lambda \in(0,1)$ we have

$$
\begin{array}{r}
(1-\lambda) q_{2}(s)+\lambda q_{4}(s)=s .\left(s^{3}+\left(\alpha_{1}+\alpha_{3}\right) s^{2}+\left(\lambda \alpha_{2}-\lambda \alpha_{4}+\alpha_{1} \alpha_{3}+\alpha_{4}\right) s-\right. \\
\left.\lambda \alpha_{1} \alpha_{4}+\lambda \alpha_{2} \alpha_{3}+\alpha_{1} \alpha_{4}\right)
\end{array}
$$

and for the third order polynomial in the bracket we have (see Introduction)

$$
a_{1} a_{2}-a_{0} a_{3}=\lambda \alpha_{1} \alpha_{2}+\alpha_{1}^{2} \alpha_{3}+\alpha_{1} \alpha_{3}^{2}+\alpha_{3} \alpha_{4}(1-\lambda)>0 .
$$

It follows that the polynomial $(1-\lambda) q_{2}(s)+\lambda q_{4}(s)$ has the root $s=0$ and three stable roots. Therefore the segment $\left[q_{2}(s), q_{4}(s)\right]$ lies on the stability boundary.

Remark 2.2 As follows from the proof of Lemma 2.1 the stability of the two segments $\left(q_{1}(s), q_{4}(s)\right)$ and $\left(q_{2}(s), q_{3}(s)\right)$ are not guaranteed.

Lemma 2.3 Consider the following polynomials

$$
\begin{aligned}
& l(s)=\left(s^{2}+c_{1} s+d\right) \cdot\left(s+c_{2}\right), \\
& l_{1}(s)=\left(s^{2}+c_{1} s+d\right) \cdot s, \\
& l_{2}(s)=\left(s^{2}+d\right) \cdot\left(s+c_{2}\right), \\
& l_{3}(s)=\left(s^{2}+c_{1} s\right) \cdot\left(s+c_{2}\right),
\end{aligned}
$$


where $c_{1}>0, c_{2}>0, d>0$. Then

1) The segments $\left[l(s), l_{i}(s)\right),\left(l_{1}(s), l_{2}(s)\right)$ and $\left(l_{2}(s), l_{3}(s)\right)$ are stable $(i=1,2,3)$,

2) The segment $\left[l_{1}(s), l_{3}(s)\right]$ belongs to the stability boundary.

Proof. We prove only stability of $\left(l_{1}(s), l_{2}(s)\right)$ : For any $\lambda \in(0,1)$ we have

$$
\begin{gathered}
(1-\lambda) l_{1}(s)+\lambda l_{2}(s)=s^{3}+\left[(1-\lambda) c_{1}+\lambda c_{2}\right] s^{2}+d s+\lambda c_{2} d \\
a_{1} a_{2}-a_{0} a_{3}=d\left[(1-\lambda) c_{1}+\lambda c_{2}\right]-\lambda c_{2} d=d(1-\lambda) c_{1}>0 .
\end{gathered}
$$

The remaining statements can be proven easily.

The polytope $\mathcal{P}$ (see (6)) has totally $\frac{n(n+1)}{2}$ edges. By Lemmas 2.1 and 2.3 among these edges the stability of

$$
\begin{array}{cl}
\frac{n(n-2)}{4} & \text { edges (if } n \text { is even), or } \\
\frac{(n-1)(n-3)}{4} & \text { edges (if } n \text { is odd) }
\end{array}
$$

are not guaranteed. The remaining

$$
\begin{array}{cl}
\frac{n(n+1)}{2}-\frac{n(n-2)}{4}=\frac{n(n+4)}{4} & \text { (if } n \text { is even), or } \\
\frac{n(n+1)}{2}-\frac{(n-1)(n-3)}{4}=\frac{n^{2}+6 n-3}{4} & \text { (if } n \text { is odd) }
\end{array}
$$

edges are either stable or belong to the stability boundary.

By using Lemmas 2.1 and 2.3 we arrive at the following mean results (Theorem 2.4 and Algorithm 2.5).

Theorem 2.4 Consider the polytope $\mathcal{P}$ (see (6)) whose $\frac{n(n+4)}{4}$ edges (if $n$ is even) or $\frac{n^{2}+6 n-3}{4}$ (if $n$ is odd) are either stable or belongs to the stability boundary. If the remaining

$$
\begin{array}{cl}
\frac{n(n-2)}{4} & \text { edges, if } n \text { is even, or } \\
\frac{(n-1)(n-3)}{4} & \text { edges, if } n \text { is odd }
\end{array}
$$

are stable or belong to the stability boundary then the inner points of this polytope are stable.

As follows from Lemmas 2.1 and 2.3, the edges, whose stability should be tested are $\left(p_{1}(s), p_{4}(s)\right), \quad\left(p_{1}(s), p_{6}(s)\right), \quad\left(p_{3}(s), p_{6}(s)\right), \quad \ldots, \quad\left(p_{2}(s), p_{3}(s)\right), \quad\left(p_{2}(s), p_{5}(s)\right)$, $\left(p_{4}(s), p_{5}(s)\right), \ldots$.

The following algorithm can be suggested for the construction of a stable polytope type (6). 


\section{Algorithm 2.5}

1) Take a factorized stable monic polynomial $p(s)$ with positive coefficients and consider $n$ boundary polynomials $p_{1}(s), p_{2}(s), \ldots, p_{n}(s)$. Construct the polytope (6). $\frac{n(n+4)}{4}$ or $\frac{n^{2}+6 n-3}{4}$ edges of this polytope are either stable or belong to the stability boundary.

2) Check the remaining $\frac{n(n-2)}{4}$ or $\frac{(n-1)(n-3)}{4}$ edges for stability by using the Segment Lemma.

3) If some segment $\left(p_{i}(s), p_{j}(s)\right)$ is not stable then for $t \in[0,1]$ consider the parallel segment

$$
\left((1-t) p(s)+t p_{i}(s),(1-t) p(s)+t p_{j}(s)\right) .
$$

Use the bisection of the interval $[0,1]$ and consider the stability of the above segment for the values $t=\frac{1}{2}, t=\frac{3}{4}, t=\frac{7}{8}, \ldots$ by using the Segment Lemma.

4) If a parallel segment is stable for $t=t_{k}$ and unstable for $t=t_{k+1}$ then choose $t=$ $\frac{t_{k}+t_{k+1}}{2}$ and continue. If additionally $t_{k+1}-t_{k}<\varepsilon$, where $\varepsilon$ is sufficiently small then stop; Take the parallel segment $\left[\left(1-t_{k}\right) p(s)+t_{k} p_{i}(s),\left(1-t_{k}\right) p(s)+t_{k} p_{j}(s)\right]$ as a new stable edge of new stable polytope $\hat{\mathcal{P}} \subset \mathcal{P}$ (see (6)).

5) Repeat the above procedure for each unstable segment $\left(p_{i}(s), p_{j}(s)\right)$ and construct a new polytope $\hat{\mathcal{P}}$ of the type (6) by using the end points of all stable segments. The inner points of the obtained polytope $\widehat{\mathcal{P}}$ are stable.

Remark 2.6 Note that the polytopes $\mathcal{P}$ and $\widehat{\mathcal{P}}$ have nonzero volumes, since the vectors $p_{1}-$ $p, p_{2}-p, \ldots, p_{n}-p$ are linearly independent.

Remark 2.7 It is well known that many stabilization problems can be reduced to the following: Given an affine polynomial family, does this family contain a stable member? Having stable polytopes the intersection problem of an affine family and a polytope is a standart linear programming problem (see $[11,12]$ for details).

Example 2.8 Let us construct a stable polytope starting from the following stable polynomial

$$
p(s)=\left(s^{2}+10 s+9\right) \cdot\left(s^{2}+s+8\right) \text {. }
$$

We have

$$
\begin{aligned}
& p_{1}(s)=\left(s^{2}+9\right) \cdot\left(s^{2}+s+8\right) \\
& p_{2}(s)=\left(s^{2}+10 s\right) \cdot\left(s^{2}+s+8\right) \\
& p_{3}(s)=\left(s^{2}+10 s+9\right) \cdot\left(s^{2}+8\right) \\
& p_{4}(s)=\left(s^{2}+10 s+9\right) \cdot\left(s^{2}+s\right)
\end{aligned}
$$

By Lemma 2.1, only the stability of the two segments $\left(p_{1}(s), p_{4}(s)\right)$ and $\left(p_{2}(s), p_{3}(s)\right)$ should be checked.

For the segment $\left(p_{2}(s), p_{3}(s)\right)$, the equation (3) has the form

$$
\left(\omega^{4}-18 \omega^{2}\right)\left(80-10 \omega^{2}\right)-\left(80-11 \omega^{2}\right)\left(\omega^{4}-17 \omega^{2}+72\right)=0
$$


and has one positive root $\omega=2.828427125$. The inequality (5) is not satisfied for this root.

For the segment $\left(p_{1}(s), p_{4}(s)\right)$, the equation (3) is

$$
\left(\omega^{4}-17 \omega^{2}+72\right)\left(9-11 \omega^{2}\right)-\left(9-\omega^{2}\right)\left(\omega^{4}-19 \omega^{2}\right)=0
$$

and has three positive roots: $\omega_{1}=1.034314101, \omega_{2}=2.59426181 \quad \omega_{3}=3$. The inequalities (4) and (5) are satisfied for these three roots. By the Lemma 1.2 the segment $\left(p_{2}(s), p_{3}(s)\right)$ is stable, whereas $\left(p_{1}(s), p_{4}(s)\right)$ is unstable.

Apply 3) and 4) of Algorithm 2.5 to the segment $\left(p_{1}(s), p_{4}(s)\right)$. Calculations give:

\begin{tabular}{|ccccc|} 
Step & $t$ & Real root(s) of eqn (3) & $\begin{array}{c}\text { Inequality (4) } \\
\delta_{1}^{e}(\omega) \delta_{2}^{e}(\omega)\end{array}$ & $\begin{array}{c}\text { Inequality (5) } \\
\delta_{1}^{o}(\omega) \delta_{2}^{o}(\omega)\end{array}$ \\
\hline & & 1.034314101 & $-1054.190<0$ & $-21.949<0$ \\
1 & 1 & 2.594261810 & $-238.007<0$ & $-147.610<0$ \\
& & 3 & 0 & 0 \\
\hline 2 & $1 / 2$ & 3.931663786 & $2347.134>0$ & $5295.146>0$ \\
\hline 3 & $3 / 4$ & 3.606562002 & $1070.466>0$ & $1885.233>0$ \\
\hline 4 & $7 / 8$ & 3.389877796 & $503.039>0$ & $736.293>0$ \\
\hline \multirow{2}{*}{5} & $15 / 16$ & 1.417206062 & $-1244.689<0$ & $-86.890<0$ \\
& & 2.184020903 & $-702.470<0$ & $-240.389<0$ \\
\hline 6 & $29 / 32$ & 3.243698456 & $246.438>0$ & $315.1387>0$ \\
\hline$\vdots$ & $\vdots$ & 3.322137183 & $372.194>0$ & $512.2813>0$ \\
\hline & & $\vdots$ & $\vdots$ & $\vdots$ \\
\hline 11 & 0.909179 & 1.848544327 & $-1105.952<0$ & $-207.790<0$ \\
& & 3.315316048 & $360.188>0$ & $492.644>0$ \\
\hline 12 & 0.908691 & 3.316459419 & $362.186>0$ & $495.901>0$ \\
\hline
\end{tabular}

After 12 steps for the value $t_{12}=0.908691$ we arrive at the stable segment $\left(\tilde{p}_{1}(s), \tilde{p}_{4}(s)\right)$, where

$$
\begin{aligned}
& \tilde{p}_{1}(s)=\left(1-t_{12}\right) p(s)+t_{12} p_{1}(s) \\
& \tilde{p}_{4}(s)=\left(1-t_{12}\right) p(s)+t_{12} p_{4}(s) .
\end{aligned}
$$

Therefore the polytope $\widehat{\mathcal{P}}=\operatorname{conv}\left\{p(s), \tilde{p}_{1}(s), p_{2}(s), p_{3}(s), \tilde{p}_{4}(s)\right\}$ is stable.

Example 2.9 Consider the following monic polynomial

$$
p(s)=\left(s^{2}+4 s+3\right) \cdot\left(s^{2}+s+5\right) \cdot(s+1) .
$$

The boundary polynomials are: 


$$
\begin{aligned}
& p_{1}(s)=\left(s^{2}+3\right) \cdot\left(s^{2}+s+5\right) \cdot(s+1), \\
& p_{2}(s)=\left(s^{2}+4 s\right) \cdot\left(s^{2}+s+5\right) \cdot(s+1), \\
& p_{3}(s)=\left(s^{2}+4 s+3\right) \cdot\left(s^{2}+5\right) \cdot(s+1) \\
& p_{4}(s)=\left(s^{2}+4 s+3\right) \cdot\left(s^{2}+s\right) \cdot(s+1) \\
& p_{5}(s)=\left(s^{2}+4 s+3\right) \cdot\left(s^{2}+s+5\right) . s .
\end{aligned}
$$

By the Lemma 1.2 the segment $\left(p_{2}(s), p_{4}(s)\right)$ is stable, but $\left(p_{1}(s), p_{4}(s)\right)$ is unstable. Algorithm 2.5 gives $t_{11}=0.946289$ and the stable segment $\left(\tilde{p}_{1}(s), \tilde{p}_{4}(s)\right)$ after 11 steps. Hence the polytope

$$
\widehat{\mathcal{P}}=\operatorname{conv}\left\{p(s), \tilde{p}_{1}(s), p_{2}(s), p_{3}(s), \tilde{p}_{4}(s), p_{5}(s)\right\}
$$

is stable.

\section{References}

[1] Bartlett, A. C., Hollot, C. V., Lin, H., "Root locations of an entire polytope of polynomials: It suffices to check the edges”, Mathematics of Control, Signals, and Systems, 1(1) (1988) : 61-71.

[2] Bhattacharyya S. P., Chapellat H. ve Keel L. H., "Robust control: The parametric approach”, New Jersey: Prentice-Hall, (1995).

[3] Barmish B. R., "New Tools for robustness of linear systems”, New York: Macmillan Publishing Company, (1994).

[4] Bialas, S., "A necessary and sufficient condition for the stability of convex combinations of stable polynomials or matrices”, Bulletin of The Polish Academy of Sciences Technical Sciences, 33(9-10) (1985) : 473-480.

[5] Aguirre, B. and Suárez, R., "Algebraic test for the Hurwitz stability of a given segment of polynomials”, Boletin De La Sociedad Matematica Mexicana, 12 (2006) : 261-275.

[6] Aguirre-Hernández, B., García-Sosa, F. R., Loredo-Villalobos, C. A., VillafuerteSegura, R., Campos-Cantón, E., "Open problems related to the Hurwitz stability of polynomials segments”, Mathematical Problems in Engineering, Volume 2018, Article ID2075903 (2018) : 8 pages.

[7] Kalinina, E. A., Smol'kin Y. A., Uteshev, A. Yu., "Stability and distance to instability for polynomial matrix families. Complex perturbations”, Linear and Multilinear Algebra, (2020) DOI: 10.1080/03081087.2020.1759500.

[8] Gayvoronskiy, S. A., Ezangina, T., Puskarev, M., Khozhaev, I., "IE control theory \& applications, 14(18) (2020) : 2825-2835.

[9] Hinrichsen, D., Pritchard, A. J., "Text in applied mathematics: vol. 48, Mathematical systems theory I. Modelling, state space analysis, stability and robustness”, Berlin, Springer-Verlag, (2005).

[10] Aguirre-Hernández, B., Frías-Armenta, M. E., Verduzco, F., "Smooth trivial vector bundle structure of the space of Hurwitz polynomials”, Automatica, 45 (2009) : 28642868. 
[11] Dzhafarov, V., Esen, Ö., Büyükköroğlu, T., "Infinite polytopes in Hurwitz stability region”, Automatica, 106 (2019) : 301-305.

[12] Dzhafarov, V., Esen, Ö., Büyükköroğlu, T., “On polytopes in Hurwitz region”, Systems \& Control Letters, 141 (2020) : 1-5.

[13] Aguirre-Hernández, B.; Frías-Armenta, M.E. and Verduzco, F., "On differential structures of polynomial spaces in Control Theory”, Journal of Systems Science and Systems Engineering, 21(3) (2012) : 372-382. 